\title{
Distribution Function of the End-to-End Distances of Linear Polymers With Excluded Volume Effects
}

\section{Jacob Mazur}

(March 9, 1965)

\begin{abstract}
The distribution function of the absolute values of chain lengths of a polymer molecule which displays the excluded volume effect cannot assume a Gaussian form. This fact follows directly from theoretical considerations based on the application of the Central Limit Theorem to the theory of Markov chains. In order to determine the exact shape of the polymer chain-end distribution function we calculated its various moments taken about the origin, and their dependence on the number of polymer segments, using a Monte Carlo technique for generating polymer chains on a lattice. The results obtained from the extrapolation of various combinations of these moments of the general form
\end{abstract}

$$
\delta_{n}=\frac{\left\langle r_{n}^{p}\right\rangle}{\left\langle r_{n}^{s}\right\rangle^{p / s}}-1 \text { for } n \rightarrow \infty
$$

are used to determine the shape of the polymer distribution function. It is found that the chain-end distribution function can be approximated by the following form:

$$
W(r) d r=\left[\Gamma\left(\frac{3}{t}\right)\right]^{-1} t \alpha^{3 / t} r^{2} \exp \left(-\alpha r^{t}\right) d r,
$$

with $t=3.2$ and $\alpha$ being a parameter, determinable from the average mean square chain-end distances.

\section{On the Incompatibility of Excluded Volume Effects With Gaussian Statistics}

The distribution of end-to-end distances in a sufficiently long, freely jointed chain is invariably a Gaussian one. This fact follows directly from the Central Limit Theorem as it is applied to the sequence of mutually independent random variables with a common distribution. (That is, each of the random variables which form a chain has the same distribution; this kind of chain is sometimes designated as a homogeneous one [1]. ${ }^{1}$ )

One can apply the Central Limit Theorem to random variables which form a homogeneous Markov chain, in order to demonstrate the fact that the Gaussian distribution law is approached in the limit for chains in which the position of a polymer unit depends on the position of the preceding one [2] as well. Real polymer chains with internal rotation around their bonds, whether free or hindered, will be accurately described by Markov chains. The classification of polymer chains as Markovian is broader than this. Thus, if one eliminates chain closures formed with a fixed number of steps (such as four-step closures on cubic lattices or six-step closures on diamond lattice), one still can apply the theory of Markov chains for these models, since the distribution of probabilities of a particular event is still determined uniquely by the preceding event,

\footnotetext{
1 Figures in brackets indicate the literature references at the end of this paper.
}

which, in this case, is a formation of a four-step or a six-step loop [3]. This description does not yet exhaust all possible cases of polymer chains which can be described by Markov chains. As has been pointed out by Montroll [4], for any polymer chain with forbidden conformations being restricted to nonself intersection of loops with $l$ intervening steps, the distribution of intrachain distances of $n$ steps will assume a limiting Gaussian form, provided that $n$ $>>$. For this reason any infinitely long polymer chain with finite-range correlations can be described as a Markov chain. The question as to whether every Markov chain will lead to a limiting Gaussian distribution is a more involved one. The restrictive conditions under which the Central Limit Theorem can be applied to a sequence of random variables forming a homogeneous Markov chain are usually satisfied for linear polymer chains as will be demonstrated. For example, one of the conditions is the nonperiodicity of the distribution of the individual segments. However, whenever a polymer chain satisfies the condition for the validity of the Central Limit Theorem, the distribution of the intramolecular distances becomes a Gaussian one, provided that the number of intervening steps is large enough.

We propose that the distribution function of polymer configurations with volume effect cannot be Gaussian even in the asymptotic case of an infinitely long chain. In other words, the application of Gaussian statistics is invalid for the case of mutually excluding random events, such as forbidden double occupancy of the same volume element of the 
polymer chain. The proof of this statement is obtained from the following heuristic, rather than from rigorous mathematical analysis: An excluded volume effect implies an existence of long-range order in a polymer chain for the simple reason that the probability distribution function of a given polymer segment is no longer independent of the initial probability distribution. (In the terminology of the theory of cooperative phenomena, a system for which there exists a correlation between the distribution of any two statistical elements, no matter how widely they are separated by other elements, is characterized by the presence of a longrange order. Therefore, excluded volume effects, defined by mutual exclusions of double occupancies of volume elements on a lattice, imply a presence of long-range correlation in a polymer chain.)

Consider a matrix $P$ of transition probabilities $p_{i j}$. The $p_{i j}$ are the probabilities for a random variable having a certain value of $\alpha_{i}$, given that the previous random variable has a value of $\alpha_{j}$. From the theory of Markov chains we know that if the matrix $P$ possesses a real nondegenerate root (or eigenvalue) $\lambda_{1}$, which has a value that is larger than the absolute value of any other root, then the probability distribution of a given random variable becomes independent of its initial distribution, provided that the number of steps is sufficiently large. In other words, the distribution of the polymer segments represented by the random variables becomes stationary, and there is no correlation between individual segments which are a long distance apart (no long-range order can exist). But we know from the Central Limit Theorem that this is precisely the condition for the Gaussian statistics to be descriptive of the distribution function of intramolecular separations. In order to complete our proof that the excluded volume effect is inconsistent with the Gaussian statistics one has to discuss the possibility of Markov chains with long range order. 'This longrange order will always be present whenever the largest root of the matrix of transition probabilities is a degenerate one [5]. Therefore, we will demonstrate that in regular polymer chains this degeneracy cannot occur as long as they are represented by Markov chains. For this purpose, the model of absorbing Markov chains is employed, since they encompass the most general type of a polymer chain in which certain chain conformations of finite number of steps are either biased or virtually excluded [3]. In absorbing Markov chains, the absorbing state represents a boundary which terminates the process of adding a step to the chain. Thus, the matrix of transition probabilities with absorbing states is stochastic. This matrix is decomposable since it can always be of the following form

$$
P=\left(\begin{array}{ll}
A_{11} & A_{12} \\
0 & A_{22}
\end{array}\right) .
$$

This matrix, being stochastic, has a largest eigenvalue equal to 1 . The matrix of transient states $A_{22}$, raised to the $n$th power represents all real conformations of an $n$-step polymer chain. By definition, its largest eigenvalue must be less than 1 in absolute value. Thus, the states of a polymer chain are decomposed into the forbidden conformations, resulting from short-range volume exclusions, and the transient states. Since we are interested only in the latter states, we demonstrate that the largest eigenvalue of the matrix $A_{22}$ is also positive and single. To do this, we employ the Frobenius theorem for matrices with nonnegative elements as follows [6]. ${ }^{2} \quad$ Any square matrix with nonnegative elements has a characteristic root which is both larger in its absolute value than any other root and is nondegenerate, provided that such a matrix cannot be decomposed, that is, no permutation matrix $T$ exists such that

$$
T A_{22} T^{-1}=P
$$

where $P$ is the matrix of the general form (1). (A permutation matrix is obtained through permutation of columns of an identity matrix.) In order to show that the matrix of transition probabilities for a chain that reached its closure in a fixed number of steps in the case of a linear homogeneous polymer is a nondecomposable one, we demonstrate the following: (1) All superdiagonal terms of such a matrix are nonzero. This follows from the fact that the superdiagonal terms represent the shortest path for the random walk to form a closed configuration (or to reach its absorbing state, which is a "point of no return"). (2) In addition, each of the columns must have at least another nondiagonal, nonzero element. This is because at least two different chain conformations should result when a step is added at random to the end of the chain. In addition, the first column (which does not possess a superdiagonal term) should have at least one nonzero element other than the top or the bottom one. Otherwise, we will encounter a periodic boundary condition, since the same chain conformation will be repeated regularly, contrary to our model for a regular polymer chain. In our other publication [3], the two bottom rows of the matrix of transition probabilities are all filled up, so that the conditions for nondecomposability of the matrix of transition probabilities are satisfied. (In regular polymer chains, one should be able to reach any permissible chain conformation from any other chain conformation by a finite number of steps.)

Another way to prove the nondecomposability of the Markov matrix with zero elements is to raise it to some power. If this operation will result in matrix having no zero elements, its nondecomposability becomes obvious. In reference [3], the matrix of eq (16) raised to the power of $l-2$, will have all its original zeros replaced by positive elements. Thus,

2 The Frobenius theorem, as quoted by most authors, deals with matrices with positive, nonzero elements [7]. This theorem has been applied in order to investigate the conditions for the absence of long-range order in certain physical
models based on Markov chains, e.g., for Ising model of ferromagnet, ordermodels based on Markov chains, e.g., for Ising model of ferromagnet, order-
disorder transitions in crystals, etc. However, there is a later Frobenius theorem [6] which deals with nonnegative matrices and which is applied here to polymer [6] which
chains. 
since there is no possibility, with simple polymer chains, to exhibit a degeneracy, or a long-range order, in their Markov-chain models, the Gaussian limiting law could obtain only when long-range excluded volume effects are absent in such chains. For example, the proof given by Montrol [4] who derived the Gaussian limiting law for any given function whose average is taken over the Markov chain, requires the existence of a single largest root of the matrix of transition probabilities. One can easily deduce from Montroll's derivation, that, if the largest root were degenerate, a Gaussian limiting law could not be obtained. ${ }^{3}$

In conclusion, we have demonstrated the following: (a) Polymers which are characterized by a chain of independent events or by homogeneous Markov chains invariably lead to Gaussian distribution of the probabilities of their lengths. This follows from the application of the Central Limit Theorem to random variables forming a statistical chain. (b) Chains which possess long-range correlation, such as an excluded volume, cannot have a Gaussian distribution for their intramolecular dimensions, even in the limiting case of an infinitely long chain. This follows from the fact that, for the distribution function of polymer dimensions to be Gaussian, the matrix of transition probabilities must be devoid of long-range order (i.e., its largest root must be nondegenerate).

Wall, Windwer, and Gans [8]; Verdier and Stockmayer [9], and Schatzki [10] have demonstrated, on the basis of Monte Carlo computations, that the distribution of end-to-end distances in a polymer chain is non-Gaussian. Similarly, Fisher and Hiley [11] have demonstrated the same thing using their chaincounting method. Thus, direct numerical computations of the distribution of polymer chain-end lengths and the theoretical considerations along the above given guidelines prove the invalidity of the application of Gaussian statistics to the polymer chains with excluded volume. It is of importance to find from numerical analysis the form of the distribution of polymer configurations with excluded volume effects, and this is the purpose of the present work.

\section{Computational Part}

It is clear from the above arguments that the excluded volume effect of a polymer chain cannot be given a theoretical treatment on the basis of theories of Markov chains. There are certain physical problems in which long-range order is accessible to theoretical treatment; e.g., critical phenomena associated with the conditions for the largest root of a Markov matrix of transition probabilities becoming a degenerate one. Unfortunately, a similar treatment of excluded volume as a long-range order does not seem to be possible in the case of a polymer chain.

\footnotetext{
${ }^{3}$ A simple statement that the Gaussian distribution is always obtained for finite Markov chains would serve our purpose, but it will be criticized as an oversimplification. We, therefore, prefer to present this more detailed proof, that such is the case with simple, linear polymer chains. Our only assumption is that all polymer chains can be described as special cases of the general class of absorbing
} chains, which were first introduced by us in reference [3].
Here, again, numerical methods rather than a theoretical analysis seem to be the only practical way for determining statistical properties of polymer chains resulting from the presence of excluded volume. Monte Carlo methods do provide a solution to the problem of estimating the mean square end-to-end distance of a polymer chain as a function of the number of steps. If one, however, tries to apply directly the same Monte Carlo calculations in order to find the polymer chain-end distribution function, one will run into technical difficulties: The "tail part" of the distribution function will show a very large scatter of data, since there will be few configurations in that part; on the other hand, near the peak of the distribution function the distances are small and one will run into the so-called "lattice effect". This effect is related to the fact that only certain discrete intramolecular separations are possible. These distances do not possess the same degeneracy, since the number of lattice sites within a given distance $r$ from a given lattice point is an irregular function of $r$ which depends on the lattice structure. The inspection of Monte Carlo data presented by Wall, Windwer, and Gans, by Verdier and Stockmayer, and by Schatzki clearly demonstrates the technical difficulties in finding the distribution of polymer configurations, except for demonstrating their non-Gaussian behavior, which is to be expected. Schatzki carried his calculations one step further by expanding the distribution function of end-to-end separations in terms of Hermite polynomials; the lowest term reduces, however, to the Gaussian distribution function for the case of no excluded volume.

Our effort will be less ambitious. By going to the case of an infinitely long chain we will establish the shape of the distribution function from certain combinations of the distance averages taken over it. By the shape of the distribution function we imply the particular term which determines its rate of decay as the distance of separation between polymer segments becomes large. If, in addition, we have another relationship that establishes the dependence of the mean-square dimension on the number of polymer segments, we can readily construct the entire polymer chain-end distribution function, which for most practical purposes should be accurate enough for the purpose of estimation of its various statistical properties.

The case of an infinitely long polymer molecule is of theoretical interest. We study this case by plotting the computed averages as functions of $1 / n$, and extrapolating the resulting curve toward $1 / n=0$. The limiting distribution function must incorporate excluded volume effects resulting from the presence of long-range correlations. As we know, incorporation of only finite-range correlations, e.g., nonself intersections of finite-sized loops in polymer chain will eventually lead to the Gaussian distribution of chain-end separations. Therefore, the true nonGaussian form of the polymer distribution function is most evident in the asymptotic case of an infinitely long chain. Moreover, the effects of the finite size 
of the excluded volume per segment and of the lattice structure are then obliterated.

Fisher and Hiley calculated the mean fourth power of end-to-end distances, $\left\langle r_{u}^{4}\right\rangle$, for nonself intersecting random walks on simple cubic and planesquare lattices, using the chain-counting method [11]. From their values of $\left\langle r_{n}^{4}\right\rangle$, they calculated the fractional variance of $r_{n}^{2}$, namely the quantity

$$
\delta_{n}=\left\langle\left(r_{n}^{2}-\left\langle r_{n}^{2}\right\rangle\right)^{2}\right\rangle /\left\langle r_{n}^{2}\right\rangle^{2}=\frac{\left\langle r_{n}^{4}\right\rangle}{\left\langle r_{n}^{2}\right\rangle^{2}}-1 .
$$

A plot of $\delta_{n}$ versus $1 / n$ is easily extrapolated to the limit of $1 / n=0$, in spite of the fact that the exact computations were carried only to the first 10 steps. For a cubic lattice, they obtained for the extrapolated fractional variance of $r_{n}^{2}, \delta_{\infty}=0.453$ (for a Gaussian distribution, $\delta_{\infty}=2 / 3$ ). We were more interested to notice the fact that, for a simple nonintersecting chain, $\delta_{n}$ reaches a limiting value rapidly; therefore, $\delta_{n}$ is practically independent of the number of steps.

A reasonable form for the polymer chain-end distribution function is of the form $W(r, n) d r=$ $C e^{-\alpha(n) r^{t} d r}$ where $C$ is determined from the normalization condition imposed on $W(r, n)$, taken over a volume of a sphere. ${ }^{4}$ If one defines

$$
\left\langle r^{p}\right\rangle=\frac{\int_{0}^{\infty} r^{p} e^{-\alpha(n) r^{t}} 4 \pi r^{2} d r}{\int_{0}^{\infty} e^{-\alpha(n) r^{t}} 4 \pi r^{2} d r}
$$

then it is evident that, for any value of $t, \frac{\left\langle r^{p}\right\rangle}{\left\langle r^{s}\right\rangle^{p / s}}$ is independent of $\alpha$, and therefore, is independent of the number of steps. However, the integrations in eq (3) should be taken not from $r=0$, but from $r=a$, where $a$ corresponds to the distance of the nearest possible approach. However, no matter how large $a$ is, in the asymptotic case of $n=\infty$, the asymptotic formula for $\delta_{n}$ should be independent of $a$. Therefore,

$$
\delta_{\infty}(p, s) \equiv \frac{\left\langle r^{p}\right\rangle}{\left\langle r^{s}\right\rangle^{p / s}}-1=\frac{\Gamma\left(\frac{p+3}{t}\right)\left[\Gamma\left(\frac{3}{t}\right)\right]^{p / s-1}}{\left[\Gamma\left(\frac{s+3}{t}\right)\right]^{p / s}}-1 .
$$

In the case of $s=2, \delta_{\infty}(p, 2)$ is identical with the fractional variance of $r^{p / 2}$, since then

$$
\delta_{\infty}(p, 2)=\frac{\int_{0}^{\infty}\left(r^{p / 2}-\left\langle r^{p / 2}\right\rangle\right)^{2} W(r, \alpha) d r}{\left\langle r^{p / 2}\right\rangle^{2}} .
$$

One would therefore expect that, if $\left\langle r^{4}\right\rangle$ and $\left\langle r^{2}\right\rangle$ were calculated for a random walk on a cubic lattice

\footnotetext{
${ }^{4}$ Recently, C. Domb [12] proposed a different form for $W(r, n)$. In the appendix of this paper these two choices for the chain-end distribution function are compared, and a more general form for $W(r, n)$, which involves two structural parameters, is introduced.
}

with unit distances between any two lattice points being forbidden, the same value of $\delta_{\infty}(4,2)$ would be obtained, as calculated by Fisher and Hiley for nonintersecting random walks on the same lattice. However, the limiting value of $\delta_{\infty}$ will be reached much slower than in the case of simple nonintersecting random walk. We therefore calculated $\left\langle r_{n}^{4}\right\rangle$ as well as $\left\langle r_{n}^{2}\right\rangle$ for a random walk on a cubic lattice with unit distances being forbidden. The special method for the Monte Carlo computations employed here and the computational details will be described elsewhere. In figure 1 the results of these computations are shown. A total number of 300,000 random walks were generated, and the computations were carried up to $n=60$. However, beyond $n=50$ the scatter of the data does not permit us to use the corresponding results.

In order to avoid crowding of data owing to the use of the reciprocal scale, the part of the curve for $22<n<40$ is redrawn in the insert on an enlarged scale. This way, the entire $\delta_{n}$ versus $1 / n$ curve as well as the detailed data which are especially needed for the accuracy of their extrapolation towards $n=\infty$ are shown. On the right-hand side of figure 1 and of the subsequent figures the values of the parameter $t$ are shown, that are calculated from the corresponding values of $\delta_{\infty}(p, s)$, as given by eq (4). Thus, the intercept of the extrapolated data of these figures with the $n=\infty$ axis leads to the correct value of the parameter $t$ of eqs (3) and (4). The various values of the parameter $t$ shown on the $n=\infty$ axis are presented in order to demonstrate the accuracy in this determination of the extrapolated value of $t$ An inspection of figure 1 not only points to the impossibility of approximating the Gaussian value of $t=2$, but to the fact that the parameter $t$ can be accurately determined to within one-tenth of a unit.

As was to be expected, the limiting value for $\delta$ is almost identical with the one reported by Fisher and Hiley $\left(\delta_{\infty}=0.443\right.$ as compared to their $\left.\delta_{\infty}=0.453\right)$.

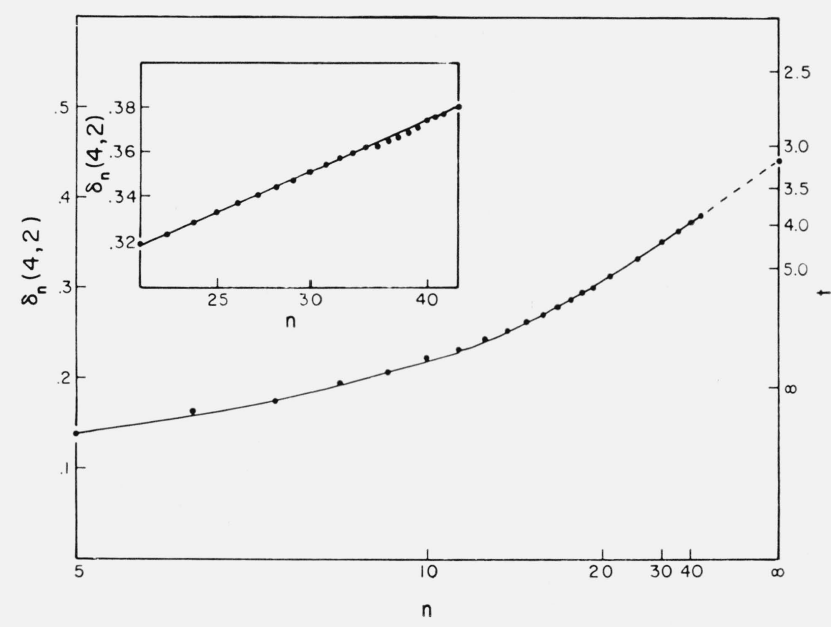

Figure 1. Plot of $\delta_{\mathrm{n}}(4,2)$, the relative fractional variance of $\mathrm{r}^{2}{ }_{\mathrm{n}}$, versus $1 / \mathrm{n}$, for random walk on a simple cubic lattice with excluded unit distance approaches. 
Moreover, the limiting value of $\delta$ is reached much slower now, owing to the fact that the distance of closest approach is considerably larger than it was for the case of simple nonintersecting chains with no other restriction being imposed on the closest possible approach between chain elements. For short chains $\delta_{n}$ is much less than its limiting value $\delta_{\infty}$, as is to be expected, considering the particular form for the distribution function. From the extrapolated value of $\delta_{\infty}(4,2)=0.443$, one finds that $t=3.2$. Therefore, for three-dimensional lattices with excluded volume, the normalized distribution function, $W(r, n) d r=3.0765 \alpha^{0.9375} r^{2} \exp \left(-\alpha r^{3.2}\right) d r$. (For two dimensional lattices, $t$ is considerably larger.) The parameter $\alpha$ is undetermined. However, it can be easily calculated from any given relationship between $\left\langle r^{p}\right\rangle$ and $n$. For example, one can replace $\alpha$ by

$$
\alpha=\left[\frac{\Gamma\left(\frac{5}{3.2}\right)}{\Gamma\left(\frac{3}{3.2}\right)\left\langle r_{n}^{2}\right\rangle}\right]^{1.6}
$$

if the relationship between $\left\langle r_{n}^{2}\right\rangle$ and $n$ is given independently.

In order to reassure ourselves that the suggested form for the distribution function serves as a good approximation, we calculated, for the same lattice model, the following averages: $\langle r\rangle,\left\langle r^{3}\right\rangle,\left\langle r^{6}\right\rangle$, and $\left\langle r^{8}\right\rangle$. Thus, $\delta_{\infty}(p, s)$ is calculated for several combinations of $p$ and $s$. In figures 2 through 5 the following fractional "averages" were computed. (If $s \neq 2, \delta(p, s)$ cannot be any more designated as a fractional variance.)

$$
\begin{aligned}
& \frac{\left\langle r^{2}\right\rangle}{\langle r\rangle^{2}}-1(p=2, s=1), \\
& \frac{\left\langle r^{6}\right\rangle}{\left\langle r^{3}\right\rangle^{2}}-1(p=6, s=3), \\
& \frac{\left\langle r^{6}\right\rangle}{\left\langle r^{2}\right\rangle^{3}}-1(p=6, s=2), \\
& \frac{\left\langle r^{8}\right\rangle}{\left\langle r^{4}\right\rangle^{2}}-1(p=8, s=4) .
\end{aligned}
$$

We notice that the results for $\delta_{\infty}(p, s)$, based on $t=3.2$ and computed from eq (4) agree all very well with the extrapolated results of figures 2 through 5 .

For further verification of this fortuitously chosen distribution function we will investigate the dependence of $\delta_{n}(p, s)$ on $n$ and on the distance of the nearest possible approach $a$, as follows:

A general expression for $\delta_{n}(p, s)$ is given in terms of incomplete Gamma Functions, $\Gamma(y, x)$,

$$
\delta_{n}(p, s)=\frac{\Gamma\left(\frac{p+3}{t}, \alpha a^{t}\right)\left[\Gamma\left(\frac{3}{t}\right), \alpha a^{t}\right]^{p / s-1}}{\left[\Gamma\left(\frac{s+3}{t}, \alpha a^{t}\right)\right]^{p / s}}
$$

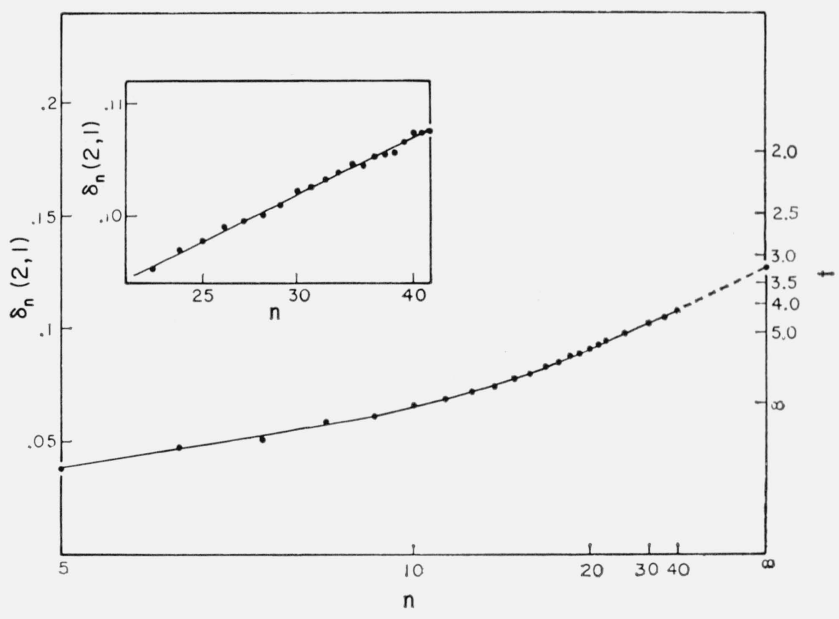

Figure 2. Plot of $\delta_{\mathrm{n}}(2,1)$ versus $1 / \mathrm{n}$.

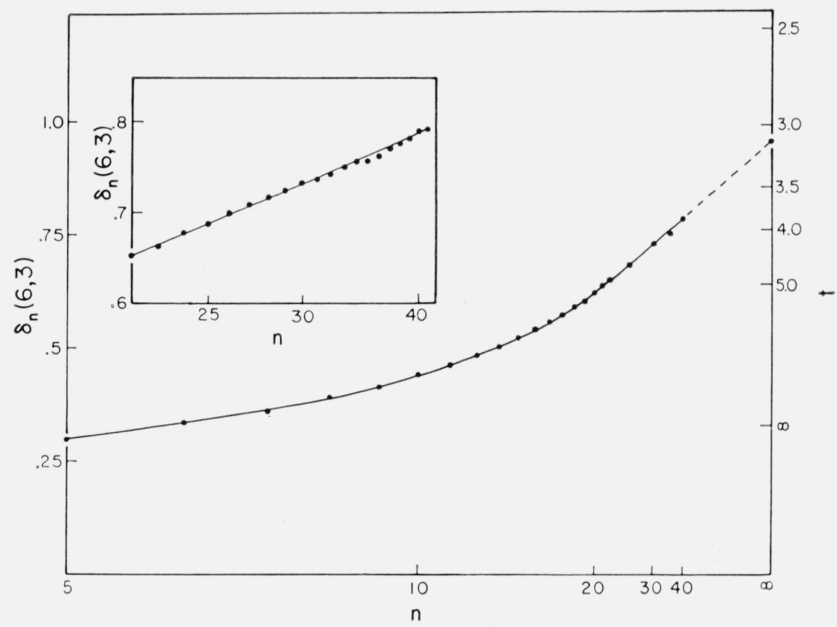

Figure 3. Plot of $\delta_{\mathrm{n}}(6,3)$ versus $1 / \mathrm{n}$.

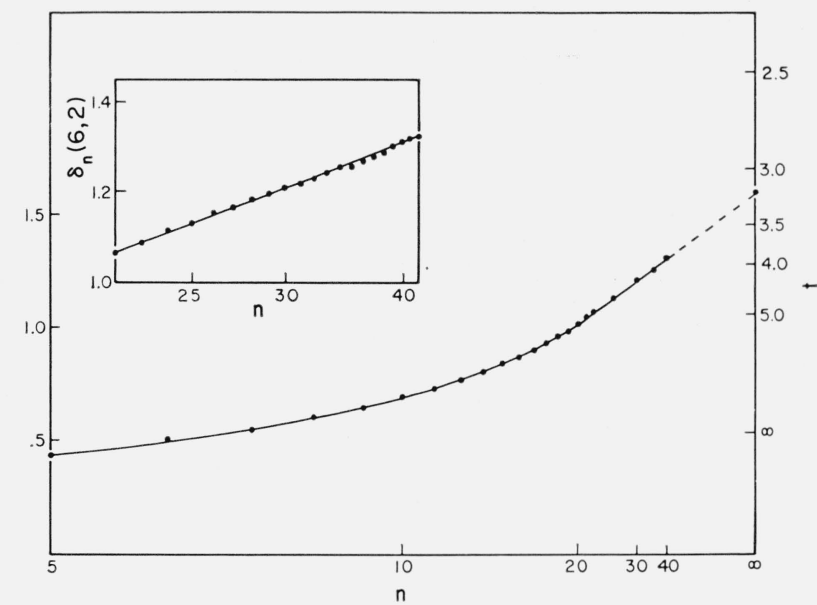

Figure 4. Plot of $\delta_{\mathrm{n}}(6,2)$ versus $1 / \mathrm{n}$. 


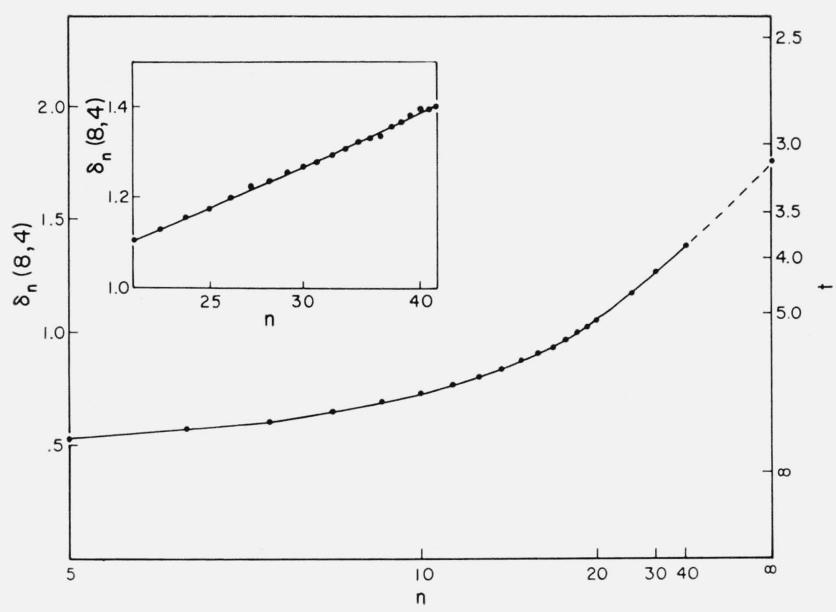

Figure 5. Plot of $\delta_{\mathbf{n}}(8,4)$ versus $1 / \mathrm{n}$.

with the following definition for $\Gamma(y, x)$,

$$
\Gamma(y, x)=\int_{x}^{\infty} e^{-t} t^{y-1} d t .
$$

The most convenient expansion for the incomplete Gamma Function with small $x$ is: $\Gamma(y, x)=\Gamma(y)-$ $y^{-1} x^{y} e^{-x} M(1,1+y, x)$ where $M(1,1+y, x)$ is a confluent hypergeometric function [13]. Since $x$, which is equal to $\alpha a^{t}$, is invariably small (except when $n$ is also small), $M(1,1+y, x)$ can be approximated by the first two terms of Kummer's Function as $M(1,1+y, x)$ $\cong 1+\frac{x}{1+y}$. Therefore,

$$
\begin{aligned}
\delta_{n}(p, s) \cong\left\{\Gamma\left(\frac{p+3}{t}\right)-\left(\frac{t}{p+3}\right) \alpha^{\frac{p+3}{t}} a^{p+3}\right. \\
\quad \times \frac{\left.\left(1-\alpha a^{t}\right)\left(1+t \frac{\alpha a^{t}}{p+3}\right)\right\}}{\left[\Gamma\left(\frac{3}{t}\right)-\frac{t}{3} \alpha^{\frac{3}{t}} a^{3}\left(1-\alpha a^{t}\right)\left(1+t \alpha a^{t}\right)\right]^{p / s-1}} \\
{\left[\Gamma\left(\frac{s+3}{t}\right)-\frac{t}{s+3} \alpha^{\frac{s+3}{t}} a^{s+3}\left(1-\alpha a^{t}\right)\left(1+\frac{\alpha a^{t}}{s+3}\right)\right]^{p / s} }
\end{aligned}
$$

Next, eq (8) can be approximated as follows:

$$
\begin{aligned}
\delta_{n}(p, s) & \cong \delta_{\infty}(p, s)\left[1-C_{1}\left(a \alpha^{1 / t}\right)^{p+3}\right] \\
& {\left[1-C_{2}(p / s-1)\left(a \alpha^{1 / t}\right)^{3}\right] \cdot\left[1+C_{3} p / s\left(a \alpha^{1 / t}\right)^{s+3}\right] }
\end{aligned}
$$

with

$$
\begin{aligned}
& C_{1}=\left[\Gamma\left(\frac{p+3}{t}\right)\right]^{-1} \cdot\left(\frac{t}{p+3}\right), \\
& C_{2}=\left[\Gamma\left(\frac{3}{t}\right)\right]^{-1} \frac{t}{3}, \\
& C_{3}=\left[\Gamma\left(\frac{s+3}{t}\right)\right]^{-1} \frac{t}{s+3} .
\end{aligned}
$$

In eq $(9)$, one notices that the deviation of $\delta_{n}(p, s)$ from its limiting value of $\delta_{\infty}$ comes primarily from the integral associated with the normalization condition. This is because the normalization integral, $4 \pi \int_{0}^{\infty} r^{2} e^{-\alpha r^{t}} d r$ involves the lowest power of $r$. Therefore, for large $n$ (i.e., for $\alpha a^{t}<<1$ ), the first-order correction to $\delta_{\infty}$ can be written as

$$
\delta_{n} \cong \delta_{\infty}\left[1-C_{2}(p / s-1)\left(a \alpha^{1 / t}\right)^{3}\right] .
$$

$C_{2}$ is here close to unity in its value. Thus, to a first approximation, $\delta_{n} / \delta_{\infty}$ for a given value of $s$ is a constant, independent of $p$, provided that $n$ is large enough for the approximations leading to eqs (8) and (9) to be valid.

In order to examine the dependence of $\delta_{n} / \delta_{\infty}$ on $p$, for given $s$, one has to consider the second dominant term in the expansion of eq (8), which is brought up by the denominator of this equation. To this approximation

$$
\delta_{n} \cong \delta_{\infty}\left[1-C_{2}(p / s-1)\left(a \alpha^{1 / t}\right)^{3}\right]\left[1+C_{3} p / s\left(a \alpha^{1 / t}\right)^{s+3}\right]
$$

and, therefore, $\delta_{n} / \delta_{\infty}$ is expected to decrease with increasing $p$ for fixed values of $s$. The following table of $\delta_{40} / \delta_{\infty}$ for $s=2$ confirms this observation.

\begin{tabular}{l|r}
\hline \hline$p$ & $\delta_{40} / \delta_{\infty}$ \\
\cline { 2 - 2 } 2 & 0.865 \\
4 & .852 \\
6 & .832 \\
8 & .809 \\
\hline
\end{tabular}

Another qualitative check of our results can be made on the basis of data presented in figures 4 and 5. These two figures represent the same value of $p$ but different values of $s$. From eq (10) it is obvious that increasing $s$ will have an effect of slightly decreasing the corresponding value of $\delta_{n} / \delta_{\infty}$. Here, again, we have $\delta_{40} / \delta_{\infty}=0.832$ for $s=2$, and $\delta_{40} / \delta_{\infty}=$ 0.827 for $s=3$, in qualitative agreement with these analytical considerations.

The dependence of $\delta_{n}$ on $n$ for the asymptotic case of large $n$ can be estimated from the following: If one assumes that $\left\langle r_{n}^{2}\right\rangle \sim A n^{\gamma}$ ( $A$ and $\gamma$ are numerical constants), then from $\alpha \propto \frac{1}{\left\langle r_{n}^{2}\right\rangle^{t / 2}}$ one obtains that $\alpha \propto \frac{1}{n^{\gamma t / 2}}$. Hence, $\delta_{n} / \delta_{\infty} \sim 1-0\left(\frac{1}{n^{2}}\right)$, for $\gamma=1.25$ and $t=3.2$, and the $\delta_{n}$ versus $1 / n$ curves should not be straight lines but somewhat concave upward in the vicinity of $1 / n=0$. Therefore, somewhat lower values for $t$ than the extrapolated value of $t=3.2$ (based on linear extrapolation of data shown in the inserts in figs. 1 through 5) might be expected.

Another aspect worth noticing is the tremendous influence of $a$, the radius of closest approach, on the 
shape of the curves in our figures. The correction term to $\delta_{\infty}(p, s)$ is proportional to at least the cube of the radius of closest approach. For this reason it is not surprising to find that in the case of excluded volume restricted to only self-intersections the limiting value of $\delta_{n}(p, s)$ is reached almost immediately, as $n$ increases, as shown by Fisher and Hiley [11], while in our case with larger $a$, it is reached considerably slower.

\section{Discussion}

The polymer distribution function for the chainend displacement to have its absolute value in the range between $r$ and $r+d r$ is given by

$$
\begin{gathered}
W(r) d r=\frac{t \alpha^{3 / t} r^{2} \exp \left(-\alpha r^{t}\right) d r}{\Gamma(3 / t)}=\frac{t}{\Gamma(3 / t)}\left[\frac{\Gamma(5 / t)}{\Gamma(3 / t)\left\langle r^{2}\right\rangle}\right]^{3 / 2} r^{2} \\
\exp \left[-\left(\frac{\Gamma(5 / t)}{\Gamma(3 / t)} \frac{r^{2}}{\left\langle r^{2}\right\rangle}\right)^{t / 2}\right] d r .
\end{gathered}
$$

For a Markov-type polymer chain, $i=2$, while for a nonself intersecting chain $t=3.2$. The latter value seems to be independent of the size of volume exclusion in the asymptotic case of an infinitely long chain. The comparison of these two representations of polymer chains is shown in figure 6 , in which $W(r)$ is plotted versus $r /\left\langle r^{2}\right\rangle^{1 / 2}$ for a Gaussian chain $(t=2)$ and for a nonself intersecting chain $(t=3.2)$. For $\left\langle r^{2}\right\rangle$, the value of $\left\langle r^{2}\right\rangle=200 l^{2}$ was taken; $l$, the length of a single polymer link is conveniently assumed to have a value of one.

The shapes of these two curves are consistent with the general trend of the effect of excluded volume on the shape of the distribution function, as pointed out by Verdier and Stockmayer [9]. Compared to the Gaussian distribution, the distribution function is deficient at low values of $r$. The tail part for large extension has a sharper cutoff, as is the case for a real polymer chain. To compensate for these effects, more polymer configurations are crowded around the mean distance. In figure 6, the position of $\langle r\rangle /\left\langle r^{2}\right\rangle^{1 / 2}$ is shown for both types of polymer chain-end distributions. In the case of a non-Gaussian chain the value of $\langle r\rangle$ agrees better with the most probable polymer configuration. This latter fact is open to experimental verification.

The fact that the polymer distribution function (or, rather, its shape) is so simply determined from certain combinations of its moments does not imply that the proposed form is the only one that could be correct. As a matter of fact, even if the single-term exponential form for the polymer distribution function were correct for the case for which it was obtained, namely, for an infinitely long polymer, this does not imply that the same function will correctly describe the entire range of polymer dimensions. It could well be that the exact polymer distribution function is built of several terms, which either converge to the form derived in this work as $n \rightarrow \infty$, or, this single-term expression for the distribu- tion function is all that is eventually left. A straightforward procedure to determine the polyme distribution function would be to calculate as many moments as it is practically possible, within the storage capacities of digital computers. Then, one can derive enough terms of a moment-generating function in order to calculate the distribution function using an inversion formula. However, we would prefer rather to test first our single-term polymer distribution function with existing experimental data, whenever applicable, and, if there is a need to do so, to seek a more exact representation.

The two computational results, our eq (13) and another relation which determines the dependence of the parameter $\alpha$ (or, the mean square distance $\left.\left\langle r_{n}^{2}\right\rangle\right)$ on the number of steps $n$ should complete the entire picture of the statistics of polymer endto-end distances. Either one of these two relationships should be regarded as a compromise rather than as a rigorous expression.

In the case of a polymer which can be adequately described as Markovian (i.e., which is devoid of excluded volume effect which results from existence of long-range intramolecular correlations), the "polymer structure index" $\gamma=1$ and $t=2$. ( $\gamma$ is defined as $\left(\left\langle r_{n+1}^{2}\right\rangle /\left\langle r_{n}^{2}\right\rangle-1\right) n$ in the limit of $n \rightarrow \infty$, assuming that such limiting value exists.) Otherwise, $\gamma$ can have any value between 1 and 2 (depending on the size of the excluded volume) and $t=3.2$. If our assumption that $t$ does not depend on the kind of a lattice, only on its dimensionality, is correct, then it is this parameter which shows, that the two classes of random walks on a lattice, the intersecting and the nonself intersecting ones, represent two distinct topological structures. A transition from one class to another class would be impossible without first breaking through the chain bonds. However, the size of the excluded volume (or the range of hardsphere potential energy for intramolecular repulsion) does not affect the topology (or the parameter $t$ ) of the chain.

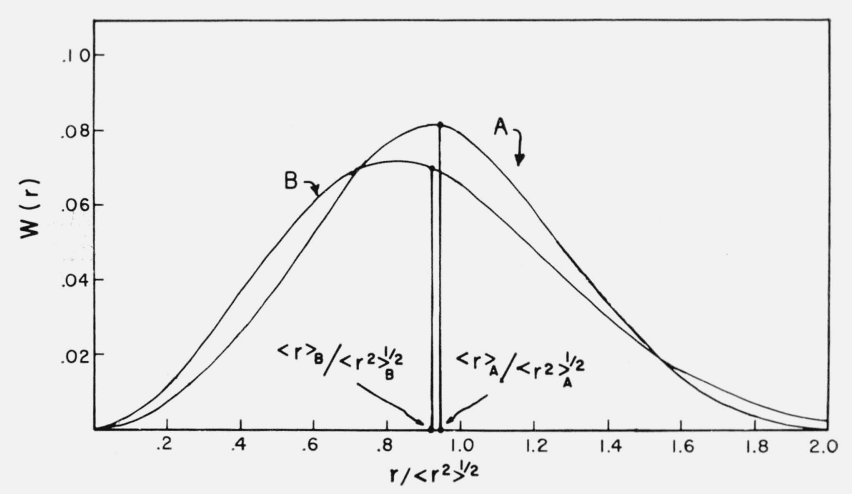

Figure 6. The chain-end distribution function for random walk on a simple cubic lattice with excluded unit-distance approaches (curve $A$ ) and for unrestricted Gaussian-type random walk on the same lattice (curve $B$ ).

In both cases $\left\langle r_{n}^{2}\right\rangle=200 \times$ unit step length. 


\section{Appendix}

Unless one considers very high moments, it is obvious that the so-called "fractional moments" $\delta_{n}$ are rather insensitive to the tail-part of the chainend distribution function. For this reason, one could expect that other forms for the radial distribution functions of the general form

$$
W(r) d r=A r^{l} \exp \left(-\alpha r^{t}\right) d r
$$

with $l \neq 2$, could lead to the correct results in terms of $\delta(p, s)$. Recently Professor Domb [12] indicated that for a simple nonintersecting random walk on a cubic lattice the general form for $W(r)$ is the above relation with $l=t$ and $t=2.5$. We checked this particular form for the distribution function, based on the proposition that $l=t$, with our data and found that for this model the value of $t=2.58$ leads to a good agreement between the computed values of $\delta(p, s)$ and these quantities as calculated from the moments of this distribution function. Moreover, if curve $A$ of figure 6 is replaced by the distribution function with $l=t$, while retaining all other parameters, the resulting curve will overlap almost exactly curve $A$, except for its tail-part. The advantage of the radial distribution function with $l=t$ lies in the fact that $W(x) d x$, the distribution of a rectangular coordinate will then assume a simple single-term exponential function with the same exponent as in the radial function, namely

$$
W(x) d x=A \exp \left[-\alpha|x|^{t}\right] d x
$$

while in our case this simple form for $W(x)$ is not obtainable [14]. As a matter of fact, a rectangular coordinate chain-end distribution function will then be given by

$$
W(x) d x=\frac{\alpha^{1 / t}}{2 \Gamma\left(\frac{3}{t}\right)} \int_{\alpha x^{t}}^{\infty} y^{\left(\frac{2}{t}-1\right)} e^{-y} d y d x
$$

assuming, as we did, that $l=2$. Of course, when $t=2$, either approach leads to the well-known oneand three-dimensional Gaussian distributions.

These considerations are illustrated in table 1 and figure 7 . In table 1 , the extrapolated values for $\delta(p, p / 2)$ are shown, together with the data calculated from the following equation for $\delta(p, p / 2)$ which is based on the general form for the chain-end distribution function given by eq (A1)

$$
\delta\left(p, \frac{p}{2}\right)=\frac{\Gamma\left(\frac{p+l+1}{t}\right) \Gamma\left(\frac{l+1}{t}\right)}{\left[\Gamma\left(\frac{\frac{p}{2}+l+1}{t}\right)\right]^{2}}-1
$$

for the following cases:
(1) $l=2, t=3.2$

(2) $l=t=2.6$

(3) $l=2.1, t=3.0$.

For comparison, the values of $\delta(p, p / 2)$ for Gaussian

\begin{tabular}{|c|c|c|c|c|c|}
\hline & $\begin{array}{l}\text { Monte Carlo } \\
\text { computation }\end{array}$ & $l=2, t=3.2$ & $l=t=2.6$ & $l=2.1, t=3.0$ & $\begin{array}{c}l=t=2.0 \\
\text { (Gaussian) }\end{array}$ \\
\hline $\begin{array}{l}\delta(2,1) \\
\delta(4,2) \\
\delta(6,3) \\
\delta(8,4)\end{array}$ & $\begin{array}{r}0.126 \\
.442 \\
.958 \\
1.735\end{array}$ & $\begin{array}{r}0.127 \\
.438 \\
.939 \\
1.696\end{array}$ & $\begin{array}{r}0.120 \\
.433 \\
.967 \\
1.816\end{array}$ & $\begin{array}{r}0.127 \\
.446 \\
.968 \\
1.770\end{array}$ & $\begin{array}{r}0.178 \\
.667 \\
1.577 \\
3.200\end{array}$ \\
\hline
\end{tabular}
distribution are also presented.

TABLE 1. Comparison of Monte Carlo computation of $\delta(\mathrm{p}, \mathrm{p} / 2)$ with values obtainted from eq (A4)

The case of $l=2.1$ and $t=3.0$ was found from computations of eq (A4) performed on a high-speed computer, as representing the best fit of results based on eq (A4) and the numerical data based on the Monte Carlo computations. This is shown in figure 7 , in which $l$ is plotted versus $t$ for each of the four "reduced" moments $\delta(p, p / 2)$. Therefore, our data justify the a priori assumption that $l=2$.

In spite of this agreement, the justification for our radial distribution function with $l=2$ is more heuristic than rigorous, owing to the uncertainty in extrapolated data based on higher moments. Thus, the total volume of all volume elements at a distance $r$ is $4 \pi r^{2} d r$, which is proportional to the number of possible positions between $r$ and $r+d r$ on a lattice. And, indeed, Wall, Windwer, and Gans [8] found that the number of possible sites on a tetrahedral lattice is approximately proportional to $r^{2}$. Thus, our distribution function retains the volume element, multiplied by a single term which represents the exponential decay of the distribution.

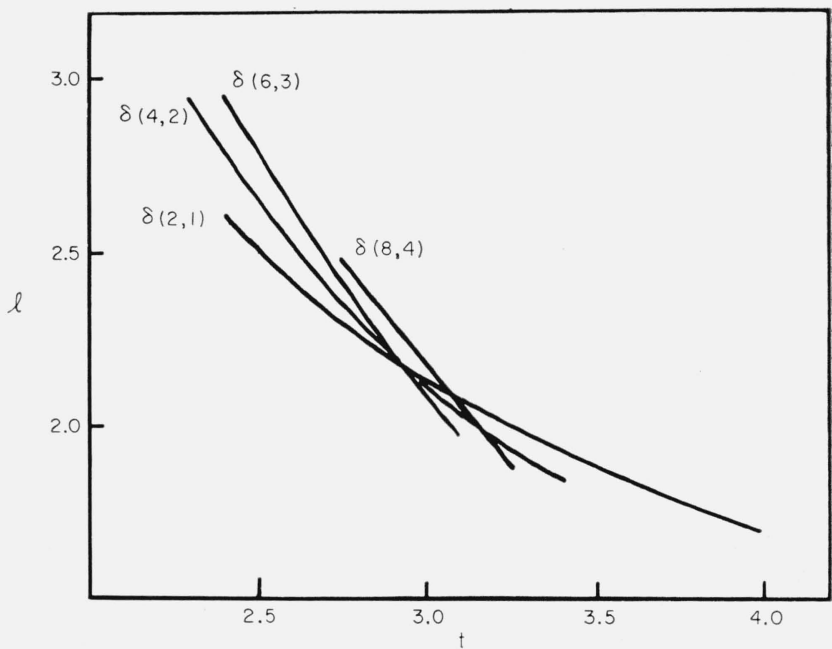

Figure 7. Graphical determination of the parameters 1 and $t$ of the chain-end radial distribution function of the general form (A1). 
However, the behavior of the distribution function as $r \rightarrow 0$ is hard to ascertain owing to the lattice effects. The indications on the basis of previous Monte Carlo calculations [8-10] are that $l>2$ represents a better description near the origin, than the case with $l=2$.

In the case of the general form of the distribution function of eq (A1) which involves two parameters, the Monte Carlo approach used here is not sensitive enough to determine a unique pair of the parameters $l$ and $t$. This is because very few samples are generated in the tail-part of the distribution which, in turn, is particularly sensitive to the exponentparameter $t$. One can improve the accuracy of our computations only if higher moments than the eighth moment are calculated. For this reason, one should generate enough samples for chains which are considerably longer than the ones generated by us, since the higher-order moments are more sensitive to the "lattice effects" than are the lower ones. We hope to undertake these computations in the future.

(Paper 69A4-355)

\section{References}

[1] M. Fisz, Probability Theory and Mathematical Statistics, ch. 6. (John Wiley \& Sons, New York, N.Y., 1963.)

[2] M. Fisz, ch. 7, p. 264. At the end of this chapter there is an extensive bibliography for conditions for the validity of the Central Limit Theorem for Markov chains.

[3] J. Mazur, J. Chem. Phys. 41, 2256 (1964).

[4] E. Montroll, Ann. of Math. Statist. 18, 18 (1947).

[5] On long-range orders in lattices see, for example, G. F. Newell and E. Montroll, Rev. Modern Physics 25, 353 (1953); J. Ashkin and W. E. Lamb, Physical Review 64, 159 (1943).

[6] G. Frobenius, Sitzungsberichte, 451, 1912; also, G. Debreu and I. N. Herstein, 21, 597 '(1953).

[7] G. Frobenius, Sitzungsberichte, 514 (1909).

[8] F. T. Wall, S. Windwer, and P. J. Gans, J. Chem. Phys. 38, 2220 (1963).

[9] P. H. Verdier and W. H. Stockmayer, J. Chem. Phys. 36, 227 (1962)

[10] T. F. Schatzki, J. Poly. Sci. 57, 337 (1962).

[11] M. E. Fisher and B. J. Hiley, J. Chem. Phys. 34, 1253 (1961).

[12] C. Domb, private communication. (See ref. [15].)

[13] Handbook of Mathematical Functions, NBS Applied Mathematics Series, pp. 262 and 504.

[14] L. R. G. Treloar, Trans Faraday Soc. 42, 77 (1946).

[15] The work mentioned in ref. 12 was in press at the time this manuscript was being prepared for print: C. Domb, J. Gillis, and G. Wilmers, Proc Phys. Soc. 85, 625 (1965). 